\title{
Experimental realization of a semiconducting quasicrystalline approximant in Al-Si-Ru system by band engineering
}

\author{
Yutaka Iwasaki, ${ }^{1, *}$ Koichi Kitahara, ${ }^{1,2}$ and Kaoru Kimura ${ }^{1,2}$ \\ ${ }^{1}$ Department of Advanced Materials Science, The University of Tokyo, 5-1-5 Kashiwanoha, Kashiwa, Chiba 277-8561, Japan \\ ${ }^{2}$ AIST-UTokyo, Advanced Operando-Measurement Technology Open Innovation Laboratory (OPERANDO-OIL), National Institute of \\ Advanced Industrial Science and Technology (AIST), 148 City Block 4, Kashiwanoha Campus, 178-4 Wakashiba, Kashiwa,
}

Chiba 277-0871, Japan

(Received 28 March 2019; published 7 June 2019)

\begin{abstract}
We found that an Al-Si-Ru cubic quasicrystalline approximant has a semiconducting band structure by performing an orbital analysis based on density functional theory. These semiconducting transport properties have been confirmed in an experimentally synthesized sample. The temperature dependences of the electrical conductivity and the Seebeck coefficient were consistent with the trends of an intrinsic semiconductor with a band gap of $0.15 \mathrm{eV}$ above $350 \mathrm{~K}$. The lattice thermal conductivity had a low value of approximately $1.0 \mathrm{~W} \mathrm{~m}^{-1} \mathrm{~K}^{-1}$ above $400 \mathrm{~K}$, which is close to the theoretical minimum.
\end{abstract}

DOI: 10.1103/PhysRevMaterials.3.061601

Since the discovery of the quasicrystal (QC) in 1984 [1], many of the unique properties of QCs have been revealed, including their crystal structures [2] and electrical properties [3]. However, the question of whether or not semiconducting or insulating QCs exist remains one of the fundamental problems to be solved in solid state physics, although the QC concept has been established as one of the categories of solid-state structures.

Semiconducting QCs have attracted attention, not only from the perspective of academic interest, but also from the viewpoint of application to thermoelectric (TE) materials to realize a direct conversion between the thermal and electrical energies. The performance of a TE material can be evaluated using the dimensionless figure of merit $z T=S^{2} \sigma T /\left(\kappa_{\mathrm{el}}+\right.$ $\left.\kappa_{\text {lat }}\right)$, where $S, \sigma, T, \kappa_{\mathrm{el}}$, and $\kappa_{\text {lat }}$ are the Seebeck coefficient, the electrical conductivity, the absolute temperature, the electronic thermal conductivity, and the lattice thermal conductivity, respectively. The highest $z T$ between QCs achieved to date is 0.26 , which is only approximately one quarter of the general target value of unity, at $500 \mathrm{~K}$ in an Al-Ga-Pd-Mn QC [4]. The main problem is that the value of $S\left(\approx 100 \mu \mathrm{V} \mathrm{K}^{-1}\right)$ is only approximately one half of that of typical practical materials. To obtain a sufficiently large $S$ at a target temperature $T$, a semiconductor with a band gap of $6-10 k_{\mathrm{B}} T$, where $k_{\mathrm{B}}$ is the Boltzmann constant, is generally required [5]. Therefore, the discovery of a semiconducting QC is necessary to enable the breakthrough of QCs for use as TE materials.

\footnotetext{
*iwasaki@phys.mm.t.u-tokyo.ac.jp

Published by the American Physical Society under the terms of the Creative Commons Attribution 4.0 International license. Further distribution of this work must maintain attribution to the author(s) and the published article's title, journal citation, and DOI.
}

While semiconductorlike properties that were attributed to a combination of the pseudogap in the density of states (DOS) and electronic weak localization were previously reported in some aluminum-transition metal (Al-TM) QCs [6-10] and quasicrystalline approximants (QCAs) $[10,11]$, a finite band gap has yet to be observed experimentally in these materials to date. There have been several theoretical works on semiconducting QCAs based on density functional theory (DFT) that have thus far contributed to the understanding of the problem of complex semiconducting QCs. Krajčí and Hafner investigated the electronic structures of hypothetical QCAs that were constructed using a model of Al-TM icosahedral QCs [12-14]. While they predicted the existence of some QCAs with semiconducting electronic structures and indicated the presence of an energy gap in QCs, these types of crystal structures have yet to be confirmed experimentally. In addition, Mihalkovič and Henley predicted that an Al-Ir QCA that exists in a thermodynamically stable phase $[15,16]$ has a semiconducting electronic structure with a band gap of $40 \mathrm{meV}$ based on a specific composition model called a 10phase model $\left(\mathrm{Al}_{22} \mathrm{Ir}_{8}\right.$ per unit cell) [17]. Structurally similar phases, which are frequently called $C, C_{1}$, and $C_{2}$ phases, are well known in binary or ternary Al-TM systems [18]. In this case, while the $C$ phase has a primitive cubic lattice, the $C_{1}$ and $C_{2}$ phases are $2 \times 2 \times 2$ superlattice structures of the $C$ phase and have body-centered- and face-centered-cubic lattices, respectively. While a semiconducting band structure was predicted for the Al-Ir $C$ phase, our previous experimental results for the TE properties showed metallic behavior because of the vacancies of the inner cluster Al sites [19]. These vacancies have a serious effect on the band structure, leading to a downward shift of the Fermi energy to the valence band and loss of the band gap.

In this Rapid Communication, we have performed an orbital analysis of the Al-Ir $C$ phase using DFT calculations to obtain a guideline to enable control of the band gap. 


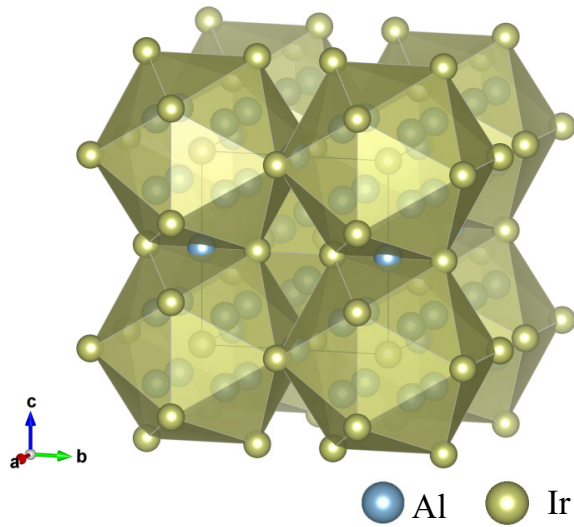

FIG. 1. Crystal structure of the calculated model of $\mathrm{Al}_{22} \mathrm{Ir}_{8}$, which was visualized using VESTA 3 [25].

Following the guideline obtained from this analysis, we found that the Al-Si-Ru $C$ phase is promising as a candidate phase for a semiconducting QCA and we synthesized a sample experimentally that shows semiconducting TE properties.

We used the WIEN2K package, which is based on the full potential augmented plane wave+local orbitals $(\mathrm{APW}+\mathrm{lo})$ method [20]. A generalized gradient approximation with Perdew-Burke-Ernzerhof parametrization (GGA-PBE) [21] was selected for the exchange-correlation energy functional. A self-consistent cycle was performed on an unshifted $10 \times$ $10 \times 10 \mathbf{k}$ mesh in the Brillouin zone using the energy cutoff constant $R_{\mathrm{MT}} K_{\max }=7.0$. Using this calculated electronic structure, we calculated the transport properties $S, \sigma$, and $\kappa_{\mathrm{el}}$ in the same manner described in Ref. [22] or see the Supplemental Material [23], based on Boltzmann transport theory within the constant-diffusion-constant approximation, which has been used frequently to explain the TE properties of QCAs [22,24].

Our calculated model of the Al-Ir $C$ phase $\left(\mathrm{Al}_{22} \mathrm{Ir}_{8}\right.$ per unit cell) shown in Fig. 1, which was visualized using VESTA 3 [25], is based on an experimental model of the Al-Ni-Ru $C$ phase that has less chemical disorder [26]. The framework for the calculated model is composed of edge-sharing $\mathrm{Ir}_{12}$ icosahedral clusters and includes an $\mathrm{Ir} @ \mathrm{Al}_{8}$ endohedral cluster that is referred to hereafter as the inner cluster. Note that the number of electrons of $\mathrm{Al}$ in the inner cluster was reduced to have the same number of valence electrons as the 10 -phase model $\left(\mathrm{Al}_{22} \mathrm{Ir}_{8}\right.$ per unit cell) using the virtual crystal approximation [27] (see Tables SI and SII and Fig. S1 in the Supplemental Material [23]).

Figures 2(a) and 2(b) show the band structure and the DOS of the Al-Ir $C$ phase, respectively. The band structure has an extremely narrow band gap of $0.02 \mathrm{eV}$, which is consistent with the previous calculations of the so-called 10-phase model in Ref. [17]. The conduction-band minimum (CBM) and the valence-band maximum $(\mathrm{VBM})$ are located at the $\Gamma$ point and the $\mathrm{X}$ point, respectively. As shown in Fig. 3, we have analyzed the corresponding Bloch states (Bloch orbitals) of the VBM and the CBM of the Al-Ir $C$ phase to find ways to enlarge the band gap, i.e., by shifting upward (downward) the CBM (VBM). The CBM consists of the Ir- $d$ orbital that exists at the vertices of icosahedral clusters. Therefore, the
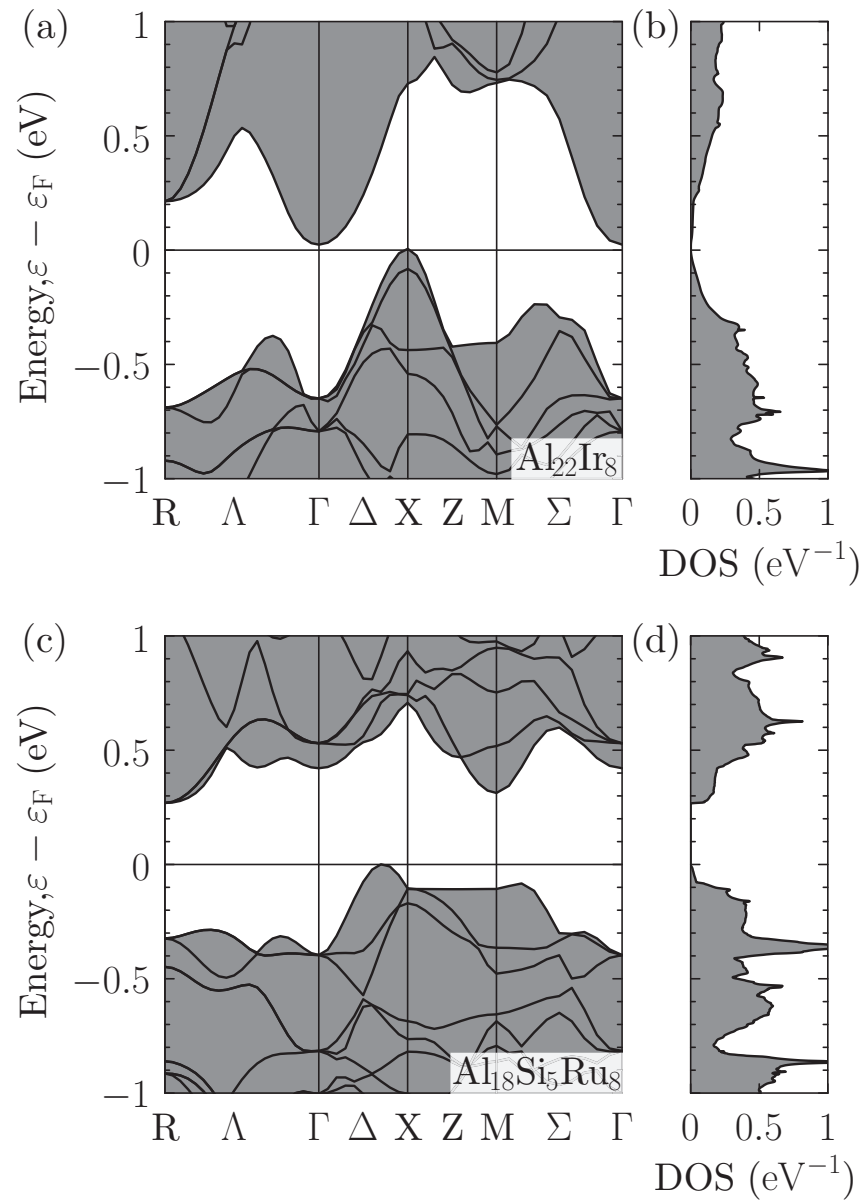

FIG. 2. Band structures and DOS per atom for the calculated models of (a), (b) $\mathrm{Al}_{22} \mathrm{Ir}_{8}$ and (c), (d) $\mathrm{Al}_{18} \mathrm{Si}_{5} \mathrm{Ru}_{8}$.

CBM energy can be shifted upward by substitution using an element with a higher $d$-orbital energy, e.g., by substituting $\mathrm{Ru}$ for Ir. In contrast, the VBM consists of $p$-like orbitals in the inner clusters. Note that these $p$-like orbitals should not be considered to be the Ir- $p$ orbitals but are the $p$ orbitals of clusters composed of Ir and the surrounding $\mathrm{Al}$, which is also confirmed in the Al-Cu-Ir $C_{2}$ phase [28]. To shift the VBM downward, it is considered effective to substitute $\mathrm{Al}$, which is the main constituent element of the inner clusters, with $\mathrm{Si}$, which has a lower orbital energy. On the basis of these findings, it is proposed that the Al-Si-Ru $C$ phase is a promising candidate semiconducting QCA. A band-structure calculation was performed for the model of the Al-Si-Ru $C$ phase $\left(\mathrm{Al}_{18} \mathrm{Si}_{5} \mathrm{Ru}_{8}\right.$ per unit cell); the structural details of this model are given in Tables SI and SII and Fig. S1 in the Supplemental Material [23]. Figures 2(c) and 2(d) show the band structure and the DOS of a hypothetical Al-Si-Ru $C$ phase $\left(\mathrm{Al}_{18} \mathrm{Si}_{5} \mathrm{Ru}_{8}\right.$ per unit cell), respectively. A finite band gap of $0.27 \mathrm{eV}$ was confirmed, thus indicating that our strategy was effective in enlarging the band gap. The CBM and VBM changed from $\Gamma$ and $\mathrm{X}$ to $\mathrm{R}$ and $\Delta$, respectively. Because the VBM is located at $\Delta$, which has lower symmetry than $\mathrm{X}$, many of the hole pockets are duplicated, leading to a band structure that is advantageous for a TE material [29]. 
(a)

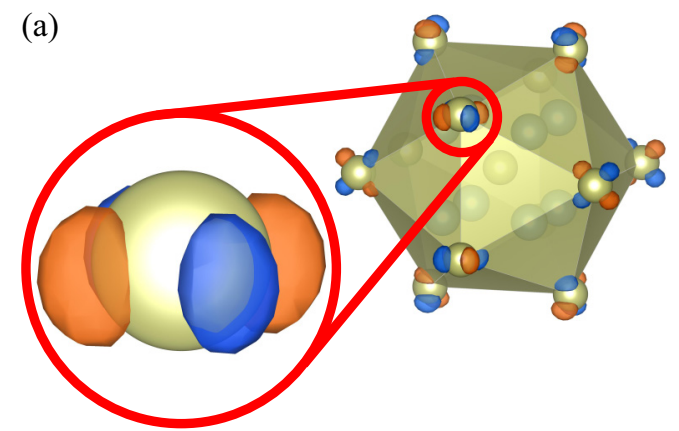

(b)

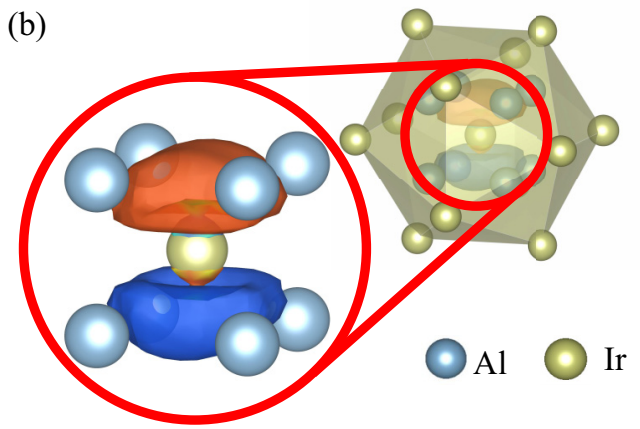

FIG. 3. Bloch states at (a) the conduction-band minimum (CBM) and (b) the valence-band maximum (VBM) for the $\mathrm{Al}_{22} \mathrm{Ir}_{8}$ model that was visualized using VESTA 3 [25]. The red and blue colors indicate the signs of the wave functions.

While the Al-Si-Ru $C$ phase was proposed to be a semiconducting phase, previous reports on the phase diagram and the formation of the $C$ phase for the ternary Al-Si-Ru system are lacking. We thus attempted to synthesize the Al-Si-Ru $C$ phase experimentally with a composition in the vicinity of $\mathrm{Al}_{18} \mathrm{Si}_{5} \mathrm{Ru}_{8} \approx \mathrm{Al}_{58.1} \mathrm{Si}_{16.1} \mathrm{Ru}_{25.8}$, which is assumed in the DFT calculations. Synthesis of the single-phase Al-Si-Ru $C$-phase sample was performed using commercial element powders of $\mathrm{Al}(4 N), \mathrm{Si}(4 N)$, and $\mathrm{Ru}(3 N)$ with a nominal composition of $\mathrm{Al}_{67.6} \mathrm{Si}_{8.9} \mathrm{Ru}_{23.5}$, where only a single-phase sample was obtained with this composition in this study (Fig. S2 in the Supplemental Material [23]). The mixed powders were pressed into disk-shaped pellets and melted using an arc-melting method under an argon atmosphere. The sample yield was more than $99 \%$. The resulting ingot was reground into powder and then sintered using the spark plasma sintering (SPS) method (SPS-515S system, Sumiseki Materials, Japan) under a uniaxial pressure of $80 \mathrm{MPa}$ in an argon atmosphere. The temperature of the specimens was then increased from room temperature to $1273 \mathrm{~K}$, and then held at that temperature for $30 \mathrm{~min}$. Because the $\mathrm{Al}-\mathrm{Si}-\mathrm{Ru} C$ phase could not be obtained after SPS, the sintered sample was then sealed in a quartz tube under an argon atmosphere and annealed at $1373 \mathrm{~K}$ for $72 \mathrm{~h}$ to enable phase formation, followed by water quenching. Note that the Al-Si-Ru $C$ phase is not stable at $1273 \mathrm{~K}$ but is stable at $1373 \mathrm{~K}$. Phase identification of the sample was then performed by powder $\mathrm{X}$-ray diffraction (XRD) measurements using $\mathrm{Cu} K-L_{2,3}$ radiation (Smart Lab, Rigaku, Japan). The lattice constants of the sample were determined via Le Bail analysis using RIETAN-FP software and a silicon powder as a line position standard (SRM640d, NIST, USA). The compositional analyses were performed using con-

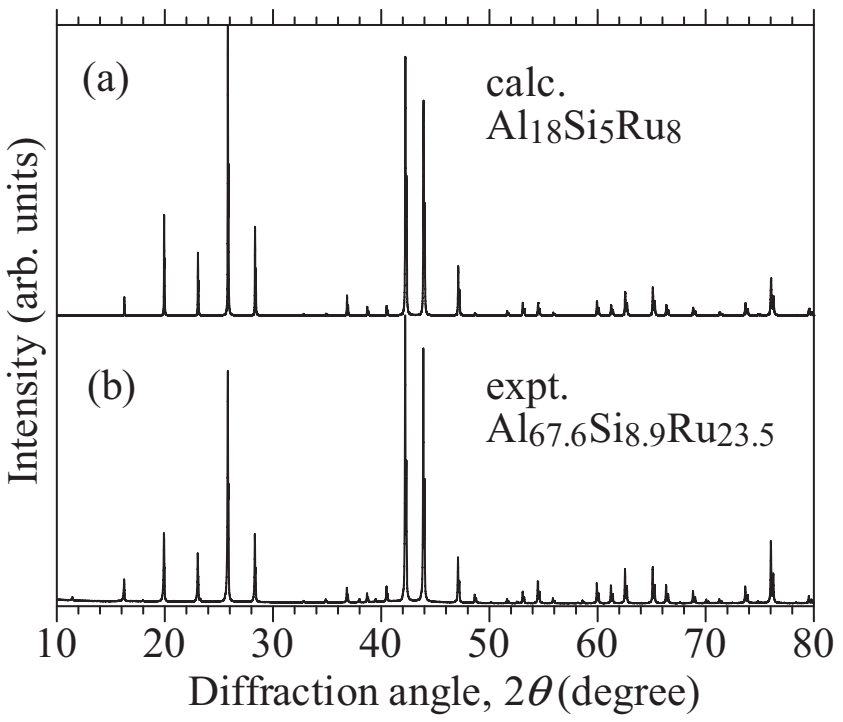

FIG. 4. XRD patterns of (a) hypothetical $\mathrm{Al}_{18} \mathrm{Si}_{5} \mathrm{Ru}_{8}$ model and (b) synthesized sample with nominal composition of $\mathrm{Al}_{67.6} \mathrm{Si}_{8.9} \mathrm{Ru}_{23.5}$.

ventional scanning electron microprobe energy-dispersive $\mathrm{x}$ ray spectroscopy (SEM-EDX) measurements (JSM-6010LA, JEOL, Japan) with elemental Al, Si, and Ru as standards for EDX. The powder and bulk densities were measured using a helium pycnometer (AccuPyc 1330, Micrometrics, USA). The relative density of the single-phase bulk sample was $98.3(6) \%$, which is sufficient for TE transport measurements. $\sigma$ and $S$ were measured under a helium atmosphere at temperatures ranging from 350 to $800 \mathrm{~K}$ using the four-probe method and the steady-state temperature gradient method, respectively (ZEM-1, Advance Riko, Japan). The total thermal conductivity $\kappa=\kappa_{\mathrm{el}}+\kappa_{\text {lat }}$ was measured using the laser flash method (TC-7000, Advance Riko, Japan) over the temperature range from 300 to $800 \mathrm{~K}$. The sound velocity $v_{\mathrm{s}}$ was measured using the ultrasonic pulse echo method (Echometer 1062, Karl Deutsch, Germany), and was then used to calculate the theoretical minimum of the lattice thermal conductivity $\kappa_{\min }[30]$.

Figure 4 shows the XRD patterns of the $\mathrm{Al}_{67.6} \mathrm{Si}_{8.9} \mathrm{Ru}_{23.5}$ sample and the hypothetical $\mathrm{Al}_{18} \mathrm{Si}_{5} \mathrm{Ru}_{8}$ model, thus showing that all the diffraction peaks were indexed with respect to the primitive cubic lattice of the lattice constant $a=0.77100(2) \mathrm{nm}$ and no secondary phase was observed. The composition obtained from the EDX analysis was $\mathrm{Al}_{67.2(3)} \mathrm{Si}_{9.2(1)} \mathrm{Ru}_{23.6(3)}$, which is almost the same as the nominal composition.

Figures 5(a)-5(d) show $S, \sigma, \kappa$, and $z T$, respectively, as functions of $T$ for the sample. $S$ at $350 \mathrm{~K}$ is more than $200 \mu \mathrm{V} \mathrm{K}^{-1}$ and is thus nearly twice the value of $120 \mu \mathrm{V} \mathrm{K}^{-1}$ obtained for the Al-Pd-Re-Fe icosahedral QC [31], which had been the highest reported value among QCs and QCAs to date. The value of $S$ decreases with increasing $T$ up to $800 \mathrm{~K}$ due to excitation of the minority carriers (electrons). In Fig. 5(a), we also show $S$ as calculated while assuming a number of holes per unit cell of 0.012 , a band gap of $0.15 \mathrm{eV}$, and an electronic diffusion constant of $0.39 \mathrm{~cm}^{2} \mathrm{~s}^{-1}$; these values are 

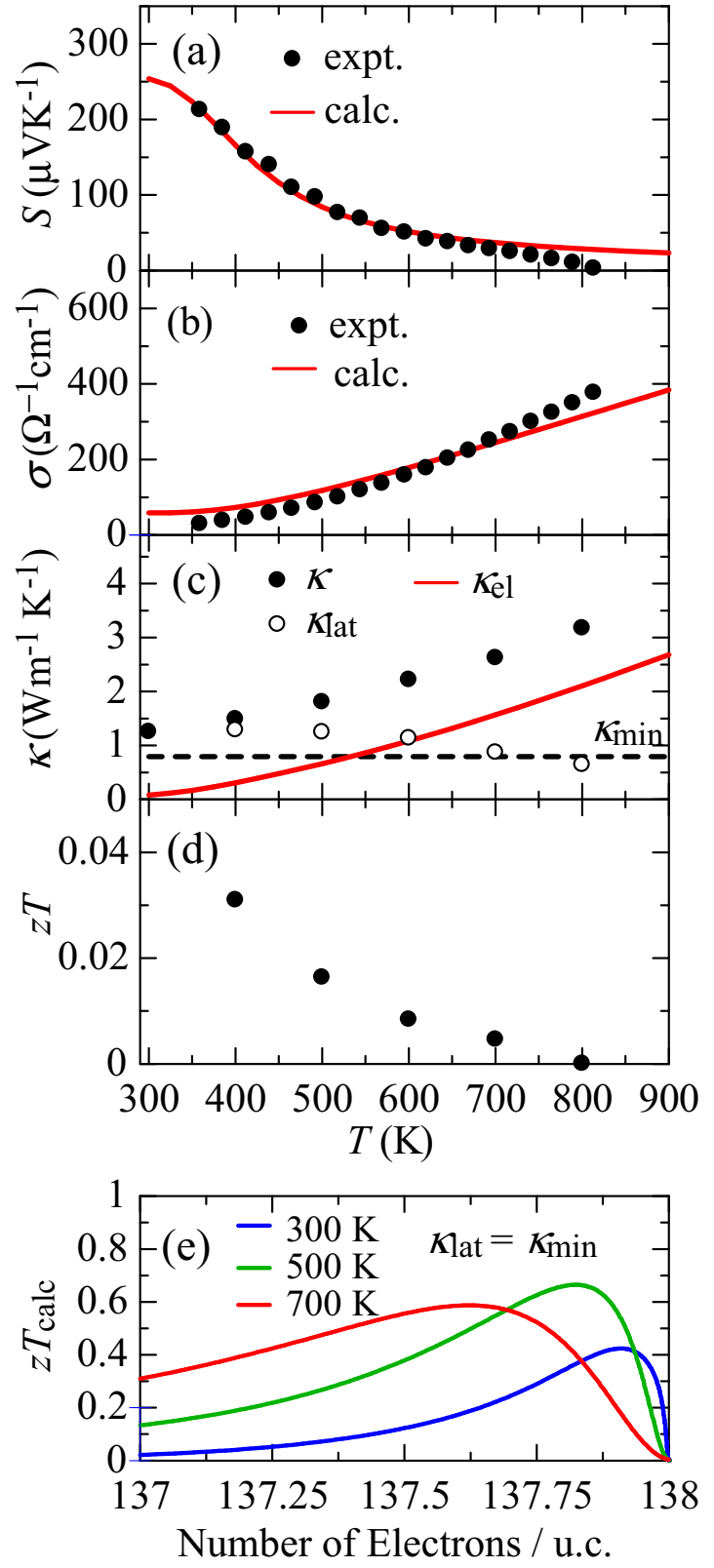

FIG. 5. Temperature dependence of (a) the Seebeck coefficient $S$, (b) the electrical conductivity $\sigma$, (c) the total and lattice thermal conductivities $\kappa$ and $\kappa_{\text {lat }}$, respectively, and (d) the dimensionless figure of merit $z T$ for the $\mathrm{Al}_{67.6} \mathrm{Si}_{8.9} \mathrm{Ru}_{23.5}$ sample. Calculated $S, \sigma$, and $\kappa_{\mathrm{el}}$ characteristics for the $\mathrm{Al}_{18} \mathrm{Si}_{5} \mathrm{Ru}_{8}$ model are also shown in (a)-(c), respectively. (e) Calculated $z T$ value $z T_{\text {calc }}$ as a function of the number of electrons per unit cell for the $\mathrm{Al}_{18} \mathrm{Si}_{5} \mathrm{Ru}_{8}$ model when assuming that $\kappa_{\text {lat }}=\kappa_{\min }$.

also used to calculate $\sigma$ and $\kappa_{\mathrm{el}}$. The calculated $S$ and $\sigma$ values are more consistent with the experimental results than those calculated using the constant relaxation time approximation. This situation is the same as that of the other QCAs [22,24]. These results indicate that the sample is a narrow-gap semiconductor with a band gap of $0.15 \mathrm{eV}$. While it is commonly recognized that the GGA-PBE functional underestimates the experimental band gap, the calculated band gap of $0.27 \mathrm{eV}$ overestimates the experimental band gap of $0.15 \mathrm{eV}$. It can be explained by the deviation of the composition between the model of $\mathrm{Al}_{18} \mathrm{Si}_{5} \mathrm{Ru}_{8} \approx \mathrm{Al}_{58.1} \mathrm{Si}_{16.1} \mathrm{Ru}_{25.8}$ and the experimental sample with lesser $\mathrm{Si}$ and $\mathrm{Ru}$ content of $\mathrm{Al}_{67.2} \mathrm{Si}_{9.2} \mathrm{Ru}_{23.6}$. As we mentioned above, the VBM consists of a $p$-like orbital in the inner cluster whose dominant elements are $\mathrm{Al}$ and $\mathrm{Si}$. When $\mathrm{Si}$ content in the experimental sample is lesser than the calculated model, the orbital energy of the inner cluster is shifted upward from the calculated VBM leading to narrowing the band gap. In contrast, the CBM consists of $d$ orbitals of the $\mathrm{Ru}$ at the icosahedral sites. When $\mathrm{Ru}$ content is lesser, which may cause site mixing of $\mathrm{Ru} / \mathrm{Al}$ or $\mathrm{Si}, \mathrm{CBM}$ is shifted downward, which also results in narrowing the band gap. For these reasons, the band-gap overestimation can be expected. However, we must clarify the crystal structure of the Al-Si-Ru $C$ phase as a future work in order to confirm this hypothesis. As shown in Fig. 5(b), $\sigma$ increases exponentially with increasing $T$, which is a typical trend in intrinsic semiconductors. The $\sigma$ at $350 \mathrm{~K}$ is approximately an order of magnitude lower than the corresponding value of the Al-Pd-Re QC [6,32-34] which was regarded as being closest to the semiconducting state to date. Figure 5(c) shows the $T$ dependences of $\kappa$ and $\kappa_{\mathrm{el}}(\equiv L \sigma T$, where $L$ is the effective Lorenz number), and $\kappa_{\text {lat }}\left(\equiv \kappa-\kappa_{\mathrm{el}}\right)$, where $L$ is obtained via DFT calculations using the same conditions that were used for $S$. $\kappa$ increases with increasing $T$ up to $800 \mathrm{~K}$ because of the increasing $\kappa_{\mathrm{el}}$. This increasing trend of $\kappa_{\mathrm{el}}$ is expected to be caused not only by the increasing $\sigma$ and $T$ but also by bipolar diffusion because $L$ has a value that is at most five times larger than the Wiedemann-Franz limit (see Fig. S3 in the Supplemental Material [23]). This bipolar diffusion behavior is consistent with the reduction of $S$ at high $T$. In contrast, $\kappa_{\text {lat }}$ exhibits a low value of approximately $1.0 \mathrm{~W} \mathrm{~m}^{-1} \mathrm{~K}^{-1}$ at room temperature because of the intrinsically complex crystal structure. Above room temperature, $\kappa_{\text {lat }}$ decreases with increasing $T$ and asymptotically approaches the theoretical minimum of $\kappa_{\min } \approx 0.76 n^{2 / 3} k_{\mathrm{B}} v_{\mathrm{s}}=$ $0.8 \mathrm{~W} \mathrm{~m}^{-1} \mathrm{~K}^{-1}$, where $n$ is the atomic density number, based on diffusion-mediated thermal transport theory [30], which gives a smaller value (at approximately 60\%) than the model proposed by Cahill et al. [35] and may thus be valid for both QCs and QCAs. Finally, we show the dimensionless figure of merit $z T$ as a function of temperature in Fig. 5(d). $z T$ decreases monotonically with increasing $T$. The maximum value of $z T$ remained relatively low at approximately 0.03 at $350 \mathrm{~K}$ because of the low $\sigma$. Furthermore, we also show the calculated $z T_{\text {calc }}$ as a function of the number of electrons per unit cell in Fig. 5(e) while assuming that $\kappa_{\text {lat }}=\kappa_{\min }$, thus indicating that further enhancement is expected from the hole doping.

In summary, we have predicted that the Al-Si-Ru $C$ phase is a semiconductor following analyses of the CBM and the VBM of the Al-Ir $C$ phase, and we also successfully synthesized an $\mathrm{Al}-\mathrm{Si}-\mathrm{Ru} C$-phase sample that exhibited semiconducting transport properties. This is an experimental realization of a semiconducting quasicrystalline approximant. The work presented here could pave the way toward a breakthrough in QC development for thermoelectric applications and the discovery of a previously undiscovered semiconducting QC. 
This work was financially supported by a KAKENHI Grant (No. JP16H04489) from the Japan Society for the Promotion of Science (JSPS). Y.I. was supported by the JSPS through the Program for Leading Graduate Schools (MERIT).
[1] D. Shechtman, I. Blech, D. Gratias, and J. W. Cahn, Phys. Rev. Lett. 53, 1951 (1984).

[2] W. Steurer, Acta Crystallogr., Sect. A: Found. Adv. 74, 1 (2018).

[3] S. Roche, G. Trambly de Laissardière, and D. Mayou, J. Math. Phys. 38, 1794 (1997).

[4] Y. Takagiwa, T. Kamimura, S. Hosoi, J. T. Okada, and K. Kimura, J. Appl. Phys. 104, 073721 (2008).

[5] J. O. Sofo and G. D. Mahan, Phys. Rev. B 49, 4565 (1994).

[6] H. Akiyama, Y. Honda, T. Hashimoto, K. Edagawa, and S. Takeuchi, Jpn. J. Appl. Phys. 32, L1003 (1993).

[7] R. Tamura, A. Waseda, K. Kimura, and H. Ino, Phys. Rev. B 50, 9640 (1994).

[8] M. Takeda, R. Tamura, Y. Sakairi, and K. Kimura, J. Phys. Soc. Jpn. 66, 1924 (1997).

[9] J. Delahaye, J. P. Brison, and C. Berger, Phys. Rev. Lett. 81, 4204 (1998).

[10] R. Tamura, T. Asao, M. Tamura, and S. Takeuchi, J. Phys.: Condens. Matter 11, 10343 (1999).

[11] R. Tamura, T. Asao, and S. Takeuchi, Phys. Rev. Lett. 86, 3104 (2001).

[12] M. Krajčí and J. Hafner, Phys. Rev. B 67, 052201 (2003).

[13] M. Krajčí and J. Hafner, Phys. Rev. B 68, 165202 (2003).

[14] M. Krajčí and J. Hafner, Phys. Rev. B 75, 024116 (2007).

[15] Y. Grin, K. Peters, U. Burkhardt, K. Gotzmann, and M. Ellner, Z. Kristallogr. - Cryst. Mater. 212, 439 (1997).

[16] M. Ode, T. Abe, H. Murakami, Y. Yamabe-Mitarai, T. Hara, K. Nagashio, C. Kocer, and H. Onodera, Intermetallics 16, 1171 (2008).

[17] M. Mihalkovič and C. L. Henley, Phys. Rev. B 88, 064201 (2013).

[18] B. Grushko and T. Velikanova, Calphad 31, 217 (2007).

[19] Y. Iwasaki, K. Kitahara, and K. Kimura, J. Alloys Compd. 763, 78 (2018).

[20] P. Blaha, G. Madsen, K. Schwarz, D. Kvasnicka, and J. Luitz, WIEN2k, An Augmented Plane Wave + Local Orbitals Programs for Calculating Crystal Properties, Vol. 1 (Vienna University of Technology Institute of Materials Chemistry, Vienna, 2017).

[21] J. P. Perdew, K. Burke, and M. Ernzerhof, Phys. Rev. Lett. 77, 3865 (1996).

[22] K. Kitahara, Y. Takagiwa, and K. Kimura, J. Jpn. Inst. Met. Mater. 82, J2017055 (2018) (in Japanese).

[23] See Supplemental Material at http://link.aps.org/supplemental/ 10.1103/PhysRevMaterials.3.061601 for detailed information of the calculated model, the way of calculating transport properties with constant-diffusion-constant approximation, nominal compositions of samples, and calculated Lorenz number.

[24] T. Takeuchi, T. Otagiri, H. Sakagami, T. Kondo, U. Mizutani, and H. Sato, Phys. Rev. B 70, 144202 (2004).

[25] K. Momma and F. Izumi, J. Appl. Crystallogr. 44, 1272 (2011).

[26] R. Simura, K. Sugiyama, S. Suzuki, and T. Kawamata, Mater. Trans. 58, 1101 (2017).

[27] A. D. Christianson, M. D. Lumsden, O. Delaire, M. B. Stone, D. L. Abernathy, M. A. McGuire, A. S. Sefat, R. Jin, B. C. Sales, D. Mandrus, E. D. Mun, P. C. Canfield, J. Y. Y. Lin, M. Lucas, M. Kresch, J. B. Keith, B. Fultz, E. A. Goremychkin, and R. J. McQueeney, Phys. Rev. Lett. 101, 157004 (2008).

[28] K. Kitahara, Y. Takagiwa, and K. Kimura, J. Phys. Soc. Jpn. 84, 014703 (2015).

[29] Y. Pei, X. Shi, A. Lalonde, H. Wang, L. Chen, and G. J. Snyder, Nature (London) 473, 66 (2011).

[30] M. T. Agne, R. Hanus, and G. J. Snyder, Energy Environ. Sci. 11, 609 (2018).

[31] J. T. Okada, T. Hamamatsu, S. Hosoi, T. Nagata, K. Kimura, and K. Kirihara, J. Appl. Phys. 101, 103702 (2007).

[32] K. Kirihara and K. Kimura, J. Appl. Phys. 92, 979 (2002).

[33] Y. Takagiwa, T. Kamimura, S. Hosoi, J. T. Okada, and K. Kimura, Z. Kristallogr. - Cryst. Mater. 224, 79 (2009).

[34] Y. Takagiwa, T. Kamimura, J. T. Okada, and K. Kimura, Mater. Trans. 55, 1226 (2014).

[35] D. G. Cahill, S. K. Watson, and R. O. Pohl, Phys. Rev. B 46, 6131 (1992). 